\title{
Prostate Acinar Adenocarcinoma, Signet Ring-Like Cell Variant
}

National Cancer Institute

\section{Source}

National Cancer Institute. Prostate Acinar Adenocarcinoma, Signet Ring-Like Cell Variant. NCI Thesaurus. Code C5535.

A morphologic variant of acinar adenocarcinoma of the prostate gland characterized by the presence of signet ring malignant cells. 